[20] G. Piccardi. Une étude sur l'influence des conditions ambiantes insolites: les tests ehimiques dans la zone aurorale et à l'intérieur de la terre. Atti I $X^{\circ}$ Convegno della Salute, 1962, Ferrare, 19-20 mai.

[21] A. Pino, L. Chrofalo. Sur les variations chronologiques de la tension superficielle du lait de chèvre entier et écrémé : recherches et comparaisons à la lumière de la méthodologie biométrique actuelle. Quaderni dell, Istituto di Zootecnia Generale dell'Università di Messina, 1957, Août.

[22] A. Pino, L. Chiofalo. Modifications de la viscosité, de la tension superficielle et de la conductibilité électrique du lait à la suite du transport. Le Lait, 1963, t. XLIII, no 421-422, p. 2.

[23] A. Pino, L. Chiofalo. Reeherches sur la corrélation entre le poids spécifique et la conductibilité électrique du lait de vache. Zootecnia e Vita, 1963, VIe année, $\mathrm{n}^{\circ} 1$.

[24] A. Pino, L. Chiofalo. Recherehes sur la conductibilité électrique du lait de chèvre. Le Lait, 1963, t. XLIII, no 429-430, p. 603.

[25] A. Pino, L. Chiofalo, F. Leonti. Importance et facteurs de hasard des données et leur importance en zootechnie. Quaderni dell'Istituto di Zootecnia Generale dell'Università di Messina, 1957, Août.

[26] E. SAvinr. La fromagerie, le lait et sa production. Hoepli, 1946, Milan.

[27] A. Tapernoux, R. Vuillaume. Viscosité du lait de vache. Le Lait, t. XVI, p. 449.

[28] S. Vianelli. Méthodologie statistique des sciences agraires. Editions Agricoles, 1954, Bologne.

[29] C. H. Withnah. La tension superficielle du lait. Un aperçu général. Journal of Dairy Science, 1959, vol. 42, 9, 1437.

\title{
FERMENTATION DU LACTOSE DANS LES DIFFÉRENTES VARIÉTÉS DE FROMAGES
}

par

\section{J. BUDSLAWSKI et K. POGORZELSKI}

Laboratoire de Chimie du Lait et des Produits Laitiers à l'École Supérieure d'Agriculture, Olsztyn, Pologne

\section{I. - Introduction}

La maturation des fromages est un processus bien compliqué dont le mécanisme n'est pas encore connu dans tous les détails. Une condition indispensable de la marche normale des étapes successives de la maturation des fromages est une fermentation régulière et rapide du lactose et des monosaccharides en dérivant par suite d'une hydrolyse dans le fromage fraîchement fabriqué $[1,2,3]$. Une marche anormale de la fermentation des sucres 
peut entraîner des défauts des fromages, c'est pourquoi la connaissance $d u$ processus de la fermentation des sucres dans toutes les phases de la fabrication des fromages est indispensable, comme aussi la connaissance du processus de leur maturation.

D'après les données bibliographiques, la fermentation du lactose dans diverses espèces de fromages marche d'une manière différente. FAGEN et al. [4] ont remarqué que, dans le fromage de Cheddar, le lactose était encore présent le $25^{\mathrm{e}}$ jour de la maturation du fromage; il en était de même avec le galactose dans le fromage fabriqué avec du lait pasteurisé, tandis que dans le fromage fabriqué avec du lait cru le galactose était présent encore après 58 jours. Le glucose se trouvait aussi dans le fromage, mais après 12 jours il disparaissait complètement.

Dans le fromage d'Emmenthal, après 10 à 12 heures déjà, Pulay [5] ne découvrait plus la présence de sucres. Dans les expériences avec les fromages Herrgard et Svecia, Sjöström [6] a montré que, s'ils étaient cuits à $44^{\circ} \mathrm{C}$, le lactose fermentait très vite ; mais le galactose restait dans les fromages jusqu'au $22^{e}$ jour de la maturation. Dans les mêmes fromages cuits à $39^{\circ} \mathrm{C}$ la fermentation du lactose s'effectuait plus lentement; mais après 2 jours les deux sucres avaient fermenté complètement. Dans aucun des fromages l'auteur n'avait découvert de glucose. NILSON et Guldstrand [7], au contraire, ont trouvé que, dans le fromage Herrgard, le lactose était présent plus longtemps que le galactose. Et Swartuing et Mattsson [8] rapportent que, dans le même type de fromage, le lactose fermente totalement en 10 à 24 jours.

Dans le fromage d'Edam, dans les conditions normales de fabrication, le lactose était présent après le salage des fromages et le galactose encore après 7 jours de maturation [9]. NAKANISHI et Tokita [10] trouvaient aussi le glucose dans ce même type de fromage.

D'après Tuckey et Sahaskabudha [11], dans le fromage de Limburg on peut trouver les trois sucres, lactose, galactose et glucose, jusqu'à la troisième semaine de maturation; mais après quatre semaines il ne reste que du lactose et du glucose.

Dans les fromages à croûte fleurie, comme dans le Camembert, on trouvait du lactose après 14 jours de maturation mais le galactose après 2 jours n'y était plus présent.

Le traitement thermique du lait et du caillé dans la chaudière semble être un facteur de grande importance, ayant une influence sur la vitesse de la fermentation du lactose. Tous les chercheurs sont d'accord sur le fait que, dans les fromages cuits à haute 
température les sucres fermentent plus lentement et, au contraire, dans les fromages cuits à basse température les sucres fermentent plus rapidement. Dans ces conditions c'est habituellement le galactose qui fermente relativement le plus lentement.

La quantité et le genre de flore microbienne des levains additionnés au lait de chaudière semblent avoir une grande influence sur la marche du processus de fermentation ; mais RAADSVELD [9] en étudiant la fermentation du lactose dans le fromage d'Edam a constaté que la quantité de levain additionné au lait n'avait qu'une faible influence, tandis que les levains mixtes fermentaient les sucres plus rapidement que les levains ne contenant qu'une espèce bactérienne.

En utilisant les levains contenant des souches de streptocoques lactiques produisant la nisine, PULAY [5] a constaté que le processus de fermentation du lactose était considérablement prolongé, et, dans ces conditions, on trouvait présents dans le fromage d'Emmenthal simultanément du lactose, du galactose et du glucose. Si dans un fromage contenant la nisine on ajoute un levain résistant à l'action de la nisine on peut, d'après LIPINSKA [12], accélérer la fermentation du lactose.

De cette courte revue des résultats obtenus par différents chercheurs, on voit qu'il y a des faits sur lesquels les auteurs sont d'accord et sur d'autres des divergences. Le comportement du glucose n'est pas clair et le mécanisme de la fermentation elle-même du lactose serait à élucider. Il semble que la vitesse de fermentation des sucres dépendrait des conditions actuelles existant dans le fromage, et surtout de la composition de la microflore.

Nous avons entrepris l'étude de la marche de la fermentation des sucres dans différents fromages à divers stades de fabrication et de maturation, en nous proposant de voir s'il y a des différences sous ce rapport dans les fromages produits dans notre Pays.

\section{II. - Matériel expérimental et méthodes}

1. On a procédé à la fabrication de fromages expérimentaux dans différentes usines. L'étude a porté sur les fromages de Tilsit, Gouda, Edam, et Emmenthal. On a fabriqué ces fromages avec du lait pasteurisé additionné d'un levain. Le fromage d'Emmenthal a été fabriqué avec du lait partiellement pasteurisé c. à d. un mélange de lait pasteurisé et de lait cru.

2. On a déterminé les sucres dans un extrait aqueux de caillé ou de fromage, préparé selon PuLAY [5] et conservé avec du toluène, par voie chromatographique sur papier Whatman 1. 
Solvant : n-butanol - pyridine - eau $(3: 2: 1,5)$;

Révélateur: solution à 2 p. 100 de chlorure de triphényltétrazol et hydroxyde de sodium $1 \mathrm{~N}(1: 1)$;

Température : $70^{\circ} \mathrm{C}$;

Temps : 5 minutes;

Eluant : solution aqueuse de pyridine et d'acide chlorhydrique, à $10 \mathrm{p} .100$.

Pour la détermination quantitative des sucres on a utilisé des solutions et des courbes standard de sucres purs.

3. On a déterminé l'acide lactique colorimétriquement selon Taylor et Clegg [13].

\section{III. - Résultats et discussion}

Les résultats de nos expériences sont montrés. dans le tableau I et, pour les différents fromages, sont représentés sur les graphiques 1 à 4. Il en ressort que la fermentation du lactose marche diffé-

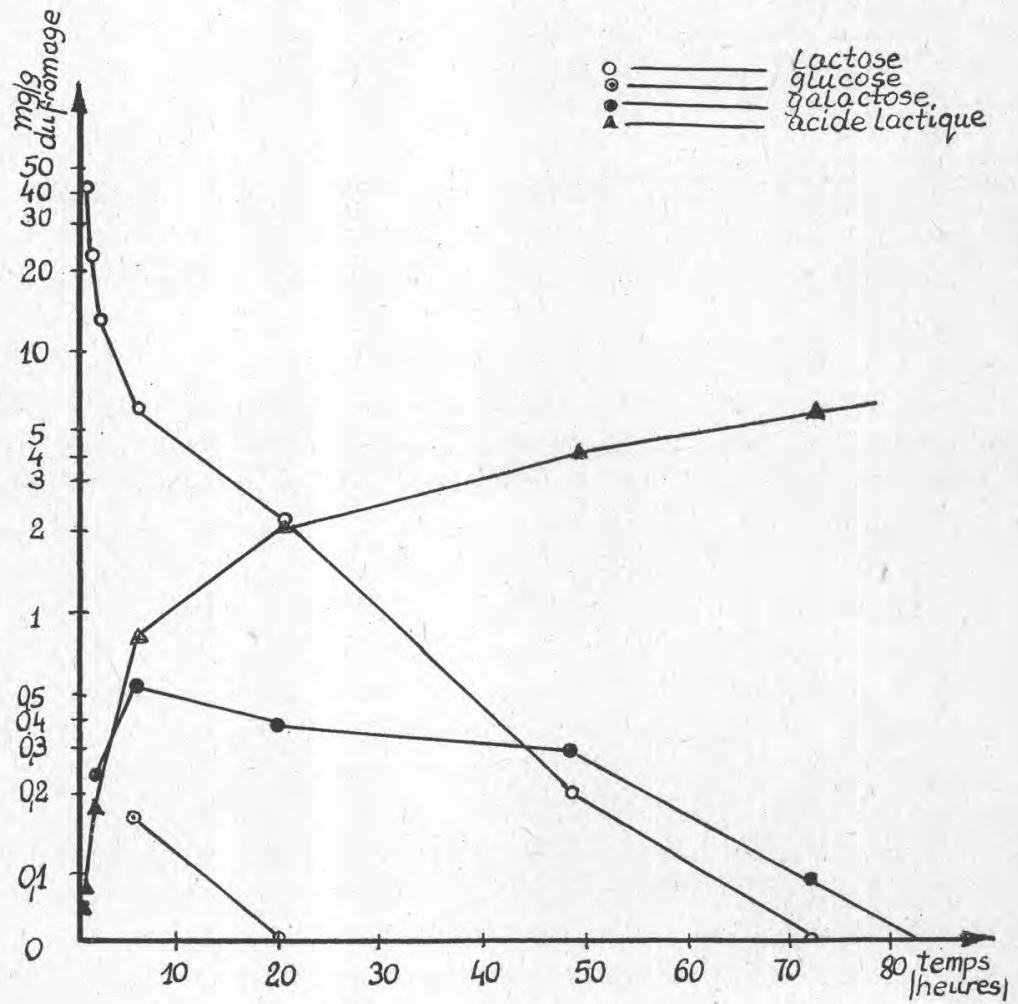

Fig. 1. - Marche de la fermentation des sucres dans le fromage de Tilsit. 


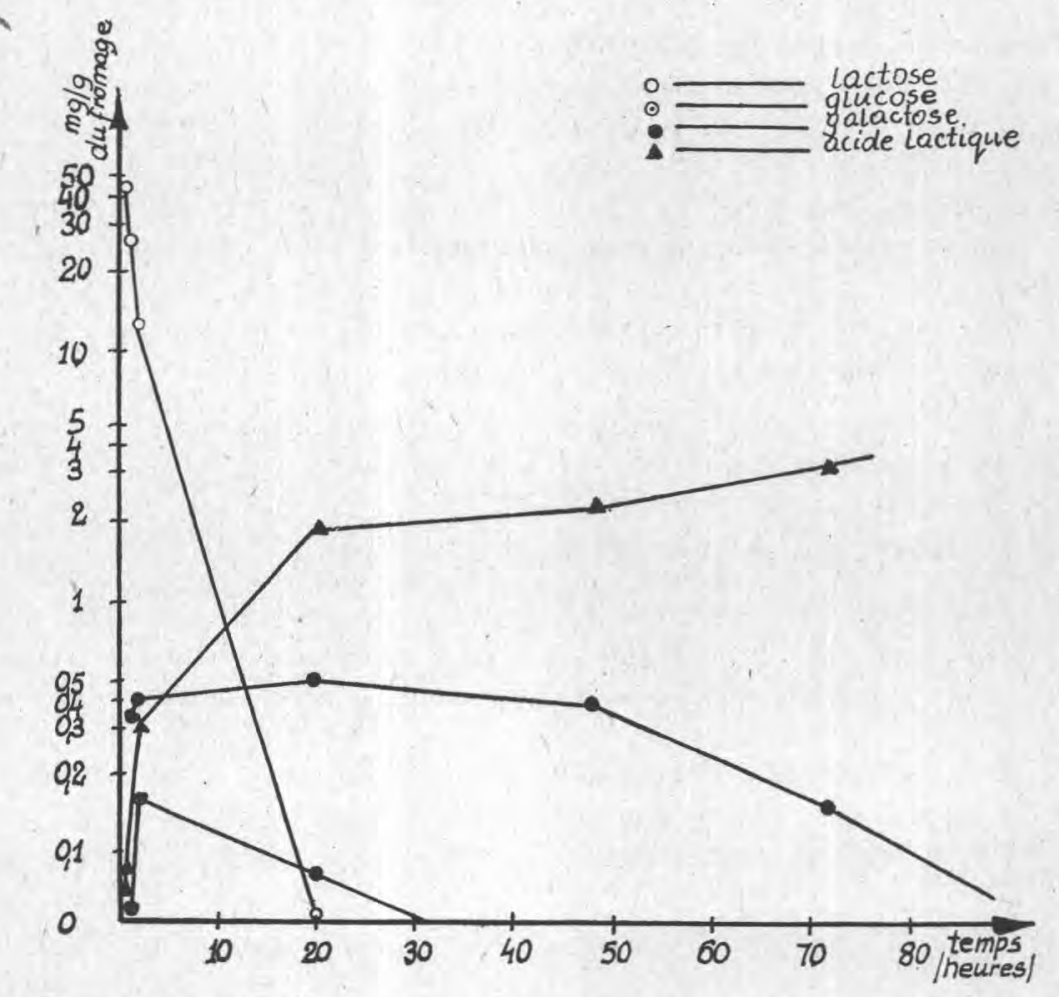

Fig. 2. - Marche de la fermentation des sucres dans le fromage Gouda.

rement dans les divers types de fromages. Dans les fromages à pâte dure et chauffés comme ci-dessus, le lactose fermente plus rapidement que dans les fromages à pâte moins dure et moins chauffés. Par exemple, dans les fromages Emmenthal et Gouda, après 20 heures, il ne reste que des traces de lactose, tandis que dans les fromages de Tilsit et d'Edam le lactose reste plus longtemps, ce qui est conforme aux observations d'autres auteurs.

La perte du lactose dans les grains de caillé et dans le fromage a lieu surtout à cause de la déshydratation et moins à cause de la fermentation. Indépendamment done du genre de fromage et de sa flore microbienne, ont une influence sur la teneur en lactose des grains de caillé et du fromage, les facteurs qui agissent sur le degré de déshydratation du caillé (évacuation du lactose avec du sérum), à savoir, l'acidité du lait, la température de cuisson, le degré d'émiéttage des grains de caillé, etc.

La teneur des fromages en galactose et en glucose est aussi différente: dans les fromages chauffés à température plus élevée 
le galactose est présent un temps moins long que dans les fromages moins chauffés; dans ces derniers le glucose apparaît aussi plus vite que dans d'autres fromages. Contrairement aux résultats d'autres auteurs [6] nos expériences montrent que le galactose fermente relativement vite, quoi que plus lentement que les autres sucres ; par exemple, dans le fromage d'Edam, après 3 jours le galactose n'était plus présent, tandis que RAadsvelt [9] le trouvait dans ce même type de fromage encore après 7 jours de maturation. La teneur la plus haute en galactose apparaît au moment de la disparition du lactose dans le fromage. A cette époque on observe aussi l'apparition du glucose, s'il y a lieu. Le galactose reste dans les fromages plus longtemps que les autres sucres.

Dans les fromages cuits à température plus élevée, une partie des microorganismes est détruite ou bien temporairement inactivée, et, dans ces conditions, le glucose, plus facilement assimilable par les microorganismes, suffit pleinement aux besoins énergé-

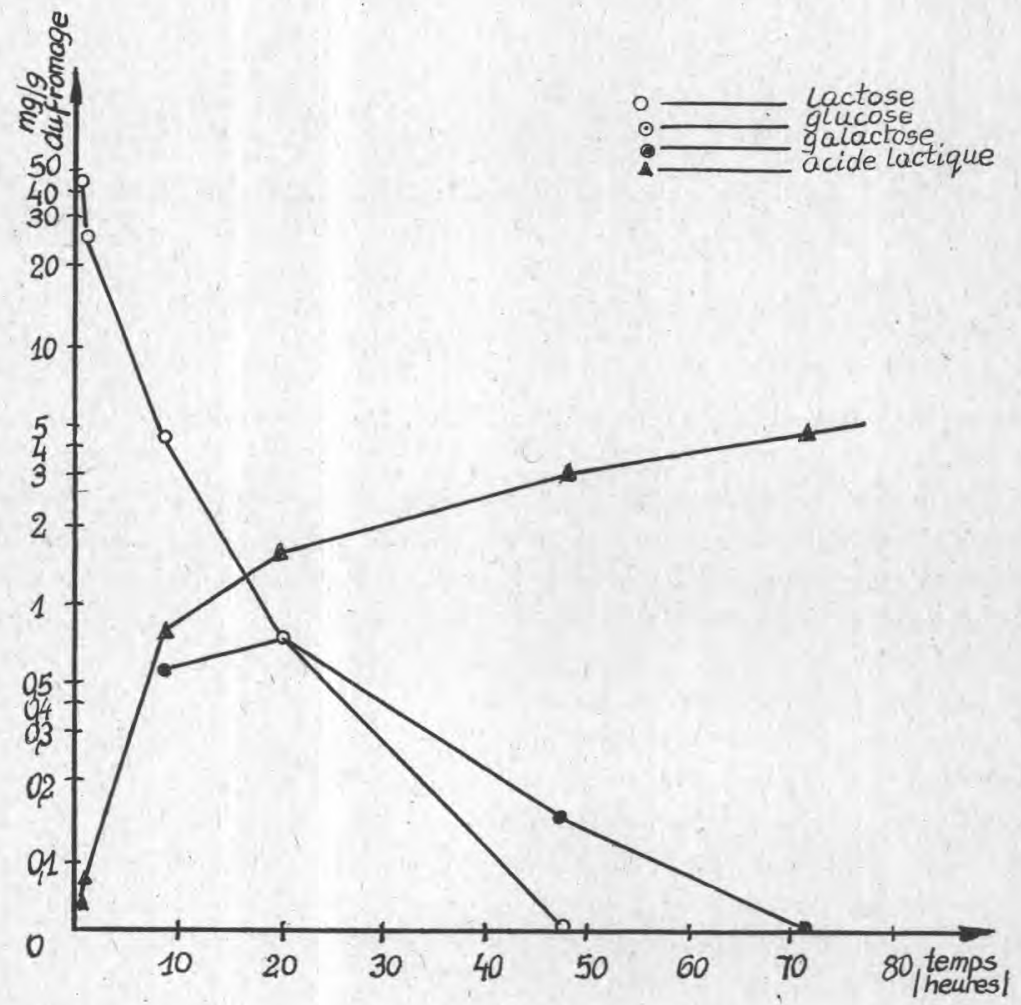

Fig. 3. - Marche de la fermentation des sucres dans le fromage Edam. 


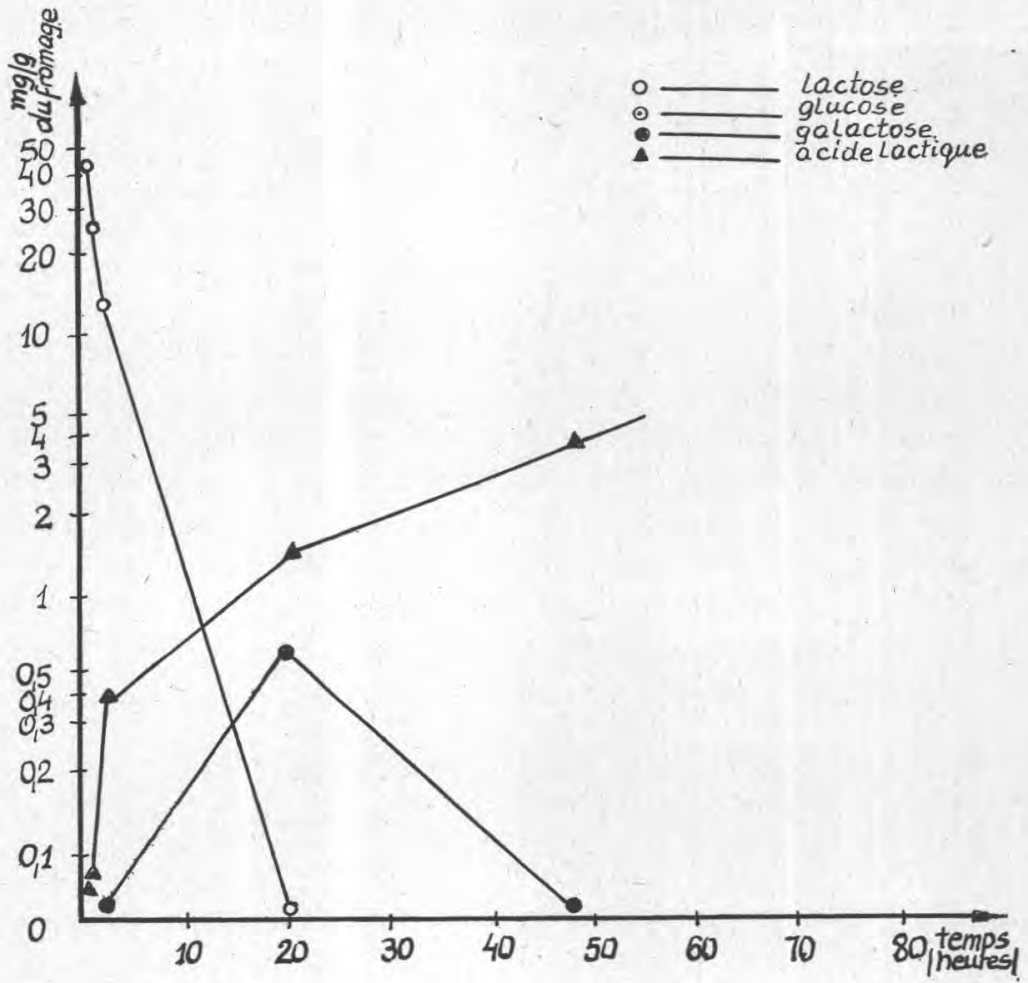

Fig. 4. - Marche de la fermentation des sucres dans le fromage d'Emmenthal

tiques de ceux-ci, et le galactose reste pour fermenter ultérieurement

Dans nos recherches, le glucose n'était détectable qu'en petites quantités dans le fromages Tilsit et Gouda (cf. tableau I) et ne se trouvait pas dans les fromages Edam et Emmenthal, tandis que Nakanishi et Tokita [10] le trouvèrent dans le fromage d'Edam. Pulay [5] a montré que les microorganismes fermentaient le glucose presque instantanément et c'est pour cela probablement que les nombreux chercheurs ne parvenaient pas à le découvrir dans des fromages pendant la maturation. Néanmoins, dans certaines espèces de fromages sa présence était constatée [4, 7]. Il en résulte que, dans des conditions déterminées, il doit y avoir des souches de ferments lactiques fermentant le glucose instantanément et des souches le fermentant lentement. Selon Pulay [5], le développement maximal des ferments lactiques a lieu au moment de la disparition du lactose dans le fromage. Les résultats de nos recher- 


\section{TABLEAU I}

LES TENEURS EN LAGTOSE, GALACTOSE, GLUGose ET AGIDE LAGTiQUe DE FROMAGES, AU GOURS DE DIFFÉRENTES PHASES DE FABRIGATION

\begin{tabular}{|c|c|c|c|c|c|c|}
\hline \multirow{2}{*}{ Fromage } & \multirow{2}{*}{ Phase de production } & \multicolumn{2}{|c|}{ Lactose } & \multirow{2}{*}{$\begin{array}{c}\begin{array}{c}\text { Galac: } \\
\text { tose }\end{array} \\
\mathrm{mg} / \mathrm{g}\end{array}$} & \multirow{2}{*}{$\begin{array}{c}\text { Glucose } \\
\mathrm{mg} / \mathrm{g}\end{array}$} & \multirow{2}{*}{$\begin{array}{c}\begin{array}{c}\text { Acide } \\
\text { lactique }\end{array} \\
\mathrm{mg} / \mathrm{g}\end{array}$} \\
\hline & & $\mathrm{mg} / \mathrm{g}$ & $\%$ & & & \\
\hline Tilsit & $\begin{array}{l}\text { Caillé, } 31^{\circ} \mathrm{C} \ldots \ldots \\
\text { Grains de caillé : } \\
\text { (cuisson), } 36^{\circ} \mathrm{C} \ldots \\
\text { (moulage), } 30^{\circ} \mathrm{C} \ldots \\
\text { Fromage après } \begin{array}{r}6 \mathrm{~h} \\
20 \mathrm{~h}\end{array} \\
48 \mathrm{~h} \\
72 \mathrm{~h} \ldots\end{array}$ & $\begin{array}{r}45,13 \\
22,25 \\
12,99 \\
5,92 \\
2,45 \\
0,21 \\
0,0\end{array}$ & $\begin{array}{r}100,0 \\
49,3 \\
28,7 \\
12,7 \\
5,4 \\
0,5\end{array}$ & $\begin{array}{l}0,0 \\
0,25 \\
0,53 \\
0,40 \\
0,32 \\
0,10\end{array}$ & $\begin{array}{l}0,0 \\
0,0 \\
0,17 \\
0,0 \\
0,0 \\
0,0\end{array}$ & $\begin{array}{l}0,044 \\
0,058 \\
0,190 \\
0,770 \\
2,368 \\
4,144 \\
6,320\end{array}$ \\
\hline Gouda & $\begin{array}{l}\text { Caillé, } 32^{\circ} \mathrm{C} \ldots \ldots \\
\text { Grains de caillé : } \\
\text { (cuisson), } 35^{\circ} \mathrm{C} \ldots \\
\text { (moulage), } 25^{\circ} \mathrm{C} \ldots \\
\text { Fromage après } 20 \mathrm{~h} \\
48 \mathrm{~h} \\
72 \mathrm{~h}\end{array}$ & $\begin{array}{c}47,56 \\
24,00 \\
11,62 \\
\text { trace } \\
0,0\end{array}$ & $\begin{array}{r}100,0 \\
50,5 \\
24,4\end{array}$ & $\begin{array}{l}0,0 \\
\\
0,33 \\
0,40 \\
0,53 \\
0,40 \\
0,17\end{array}$ & $\begin{array}{l}0,0 \\
\text { trace } \\
0,17 \\
0,08 \\
0,0 \\
0,0\end{array}$ & $\begin{array}{l}0,006 \\
0,074 \\
0,332 \\
2,014 \\
2,368 \\
3,552\end{array}$ \\
\hline Edam & $\begin{array}{l}\text { Caillé, } 31^{\circ} \mathrm{C} \ldots \ldots \\
\text { Grains de caillé } \\
\text { (cuisson), } 39^{\circ} \mathrm{C} \ldots \\
\begin{array}{r}\text { (moulage) } \ldots \\
\text { Fromage après } \quad 8 \mathrm{~h} \\
20 \\
20 \mathrm{~h} \\
48 \mathrm{~h} \\
72 \mathrm{~h}\end{array}\end{array}$ & $\begin{array}{l}48,0 \\
26,35 \\
- \\
4,81 \\
0,68 \\
0,0\end{array}$ & $\begin{array}{r}100,0 \\
54,9 \\
- \\
10,0 \\
1,4\end{array}$ & $\begin{array}{l}0,0 \\
0,0 \\
- \\
0,53 \\
0,68 \\
0,17 \\
0,0\end{array}$ & $\begin{array}{l}0,0 \\
-\overline{0} 0 \\
0,0 \\
0,0 \\
0,0\end{array}$ & $\begin{array}{c}0,012 \\
0,074 \\
- \\
0,772 \\
1,576 \\
3,078 \\
4,500\end{array}$ \\
\hline $\begin{array}{c}\text { Em- } \\
\text { menthal }\end{array}$ & $\begin{array}{l}\text { Caillé, } 32^{\circ} \mathrm{C} \\
\text { Grains de caillé } \\
\text { (cuisson), } 52^{\circ} \mathrm{C} \ldots \\
\text { (moulage), } 35^{\circ} \mathrm{C} \ldots \\
\text { Fromage après } 20 \mathrm{~h} \\
48 \mathrm{~h} \\
72 \mathrm{~h} \\
72\end{array}$ & $\begin{array}{c}24,61 \\
12,48 \\
\text { trace } \\
0,0\end{array}$ & $\begin{array}{r}100,0 \\
\\
53,4 \\
27,1\end{array}$ & $\begin{array}{c}0,0 \\
\text { trace } \\
0,60 \\
\text { trace } \\
0,0\end{array}$ & $\begin{array}{l}0,0 \\
0,0 \\
0,0 \\
0,0 \\
0,0\end{array}$ & $\begin{array}{c}0,070 \\
0,086 \\
0,414 \\
1,580 \\
3,540 \\
-\end{array}$ \\
\hline
\end{tabular}

ches sont conformes aux observations de cet auteur, puisque la plus grande augmentation de la teneur en acide lactique, dans tous les cas, coïncide avec la disparition du lactose. En même temps on 
observe la plus haute teneur des fromages en galactose et, éventuellement, en glucose.

L'augmentation de l'acidité, exprimée en acide lactique, au début du traitement thermique du caillé dans la chaudière est semblable dans tous les fromages; mais au bout de 20 heures la teneur en acide lactique des fromages Tilsit et Gouda devient plus haute que celle des fromages Edam et Emmenthal. Dans les fromages cuits à une température plus élevée l'augmentation de l'acidité est plus lente que dans les fromages cuits à température basse ; mais dans les heures qui suivent, l'augmentation de l'acidité croît plus rapidement dans le fromage de Tilsit et le plus lentement dans le fromage de Gouda.

\section{IV. - Conclusion}

Les résultats de nos recherches montrent que dans les fromages la fermentation du lactose et des sucres en dérivant ne marche pas de la même manière. On voit que le glucose fermente le plus rapidement et le galactose le plus lentement, ce qui est conforme aux résultats d'autres auteurs. Dans tous les fromages étudiés, le lactose et le glucose après 24 à 48 heures, et le galactose après 72 heures, pratiquement, ont fermenté complètement. Cela veut dire qu'au bout de trois jours les fromages sont exempts de matériel susceptible de fermenter, ce qui est favorable pour la marche correcte de leur maturation ultérieure. Puisque la fermentation des sucres dans les fromages ne marche pas d'une manière uniforme, et dépend visiblement de la composition de la flore microbienne présente dans le fromage, il serait indiqué d'étudier individuellement la dynamique de la fermentation des sucres à l'aide des différentes souches de ferments lactiques.

\section{Summary}

The preliminary studies on the ripening process of cheese concentrated on the course of the lactose fermentation in cheeses most often produced in Poland, e.g. Tilsit, Gouda, Edam and Emmentaler cheeses.

It follows from the obtained data that lactose fermentation is practically over after 24 to $48 \mathrm{hrs}$. and that after $72 \mathrm{hrs}$. there are also other sugars, which means that cheese contains no other fermentative material and which is of great importance in the cheese ripening process. 


\section{BIBLIOGRAPHIE}

[1] A. Tоневотавеv. Biokhimitcheskye osnovy sozrevanya syrov, Moscou, 1959.

[2] E. Pijanowski. Zarys chemii i technologii mleczarstwa. Varsovie, 1956.

[3] J. Kurmann. Dtsche Molkerei Ztg., 78, 1146, 1957.

[4] H. F $\triangle$ Gen, J. Stine et R. Hussong. J. Dairy Sci, 35, 779, 1952.

[5] G. PUlay. XIVe Congrès Int. Laiterie, 2, 432, 1956.

[6] G. SృöströM. XIVe Congrès Int. Laiterie, 3, 562, 1956.

[7] R. Nilson et M. Guldstrand. XVe Congrès Int. Laiterie, 3, 1773, 1959.

[8] P. Swarting et S. Matisson. XiII Congrès Int. Laiterie, 2, 615, 1953.

[9] C. W. RaAdsveld. Versl. Ned. Inst. Zuivelonderzoek, no 27, 1957.

[10] T. Nakanishi et F. Tokita. J. Agr. Res., 9, 37, 1958.

[11] S. Tuckey et M. SaHasrabudha. J. Dairy Sci., 39, 918, 1956.

[12] E. Lipinska. Prace Inst. Przem. Mlecz. Varsovie, 8, 1, 1961.

[13] P. TAYlor et L. Clegg. J. Dairy Res., 25, 32, 1958.

Cette étude fait partie du travail exécuté avec les fonds de United States Department of Agriculture, Agricultural Research Service. (FG-PO-151). Les auteurs expriment leur vive reconnaissance à cet Organisme.

\section{MESURE DE LA TENSION SUPERFICIELLE DE QUELQUES LAITS \\ AU TENSIOMĖTRE A ANNEAU \\ DE LECOMTE DU NOUY (1)}

par

\section{A. CALANDRON}

Ecole Nationale Supérieure

Agronomique de Rennes.

\section{GRILLET}

Faculté des Sciences

de Rennes

\section{Remarques préliminaires}

Afin d'éviter les erreurs de mesure de la tension superficielle dues soit aux variations de la température du liquide à mesurer, soit aux vapeurs ou gaz de l'atmosphère, nos essais furent effectués dans un grand laboratoire de physique ne servant, au cours de la journée, qu'aux expériences décrites et seulement occupé par les auteurs de cette Note.

(1) Bull. Soc. sc. Bretagne. (Bulletin 37, paru en avril 1964.) 